\title{
Robust Tracking of a Mobile Beacon using Time Differences of Arrival with Simultaneous Calibration of Receiver Positions
}

\author{
Johannes Wendeberg, Jörg Müller, Christian Schindelhauer, Wolfram Burgard \\ Department of Computer Science, University of Freiburg, Germany, \\ \{wendeber, muellerj, schindel, burgard\}@informatik.uni-freiburg.de
}

\begin{abstract}
Localization based on time differences of arrival (TDOA) has turned out to be a promising approach when neither receiver positions nor the positions of signal origins are known a priori. In this paper, we consider calibration-free tracking of a mobile beacon using TDOA, i.e., the positions of the receivers are not given. We propose a probabilistic formulation using a particle filter to simultaneously localize the signal beacon and the receivers. Our method is robust against measurement outliers and incorrect initialization. This is achieved through a probabilistic sensor model for TDOA data which explicitly considers the measurement uncertainty and takes into account disproportional errors caused by measurement outliers. For the reliable initialization of the particle filter, we apply an iterative optimization approach to multiple subsets of TDOA data, where the best solution is implicitly selected by appropriate weighing of the sensor model. We verify the robustness of our approach in extensive experiments in a spacious indoor environment by an ultrasound beacon moving on various trajectories. We demonstrate that our approach ensures a proper initialization of the particle filter and provides accurate position estimates for the signal beacon and the receivers even in case of measurement outliers. Compared to position references of an optical motion capture system we achieve mean position errors below 5 centimeters.
\end{abstract}

\section{INTRODUCTION}

The continuous rise and the ubiquitous availability of technology in every-day life and in industry has led to an increasing demand for location awareness in indoor and outdoor environments. While the GPS system has become a reliable assistant in the open field and in outdoor navigation, the disposition of localization systems begins to shift towards indoor scenarios.

Localization based on the runtime of acoustic signals is a promising technology, as the propagation velocity of sound or ultrasound signals is almost constant. Localization systems based on time differences of arrival (TDOA) have the substantial benefit that the target to be tracked only has to emit a signal and requires no cooperation with the receivers. The construction of the target is simple and inexpensive as no communication and computational power is required. Only the stationary receivers require a communication network, e.g., WiFi, to exchange TDOA information.

In this paper, we consider the problem of estimating the trajectory of a moving signal beacon and the position of multiple stationary receivers distributed in the environment. We require no interaction between the beacon and the receivers, except that the short signal bursts emitted by the beacon are detected

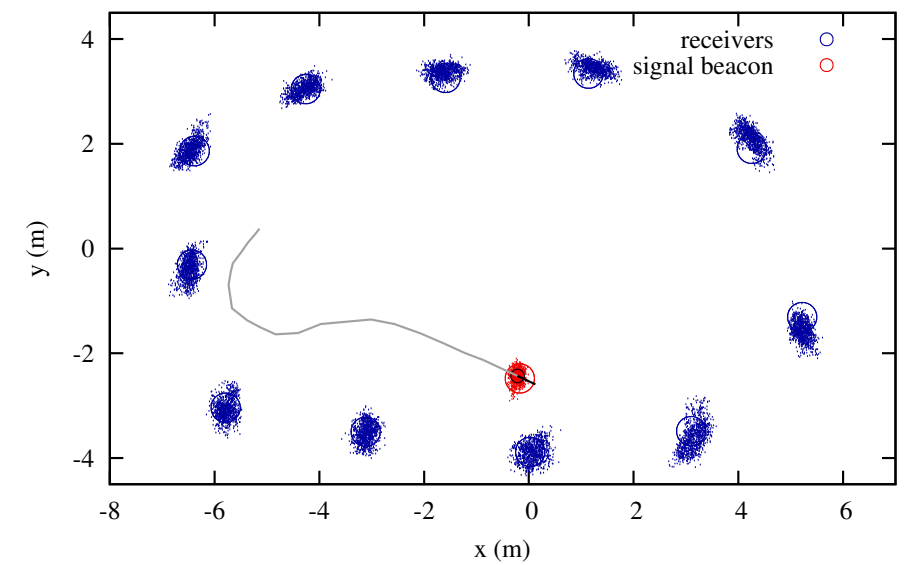

Fig. 1. A visual representation of the state estimate of the particle filter. The receivers are shown in blue and the signal beacon in red. The ground truth positions are depicted as blue and red circles, respectively.

and distinguished by the receivers. The receiver measurements of the arrival times of the individual bursts are used as the only input to the estimation process.

We present a robust approach for the localization of a moving signal beacon with simultaneous calibration of the receiver positions using probabilistic state estimation in a particle filter. Our algorithm takes into account the continuous trajectory of the signal beacon and can therefore reliably estimate its trajectory and recover its position even in case of temporary signal loss. Furthermore, our probabilistic sensor model for TDOA data explicitly considers the measurement uncertainty and takes into account disproportional errors caused by measurement outliers. In contrast to most TDOA localization systems, which assume a Gaussian distribution of the measurement errors, our approach is robust to effects like multipath signal propagation (echoes) which often occur in indoor environments. We initialize the particle filter estimate through multiple iterative optimization attempts on randomly chosen subsets of the available measurement data. Hence, our algorithm is robust to measurement outliers also in the initialization phase. Due to the weighting of the particles according to our TDOA sensor model, the particle filter implicitly selects the correct initialization hypothesis and reliably converges to the true configuration. Additionally, we detect the proper 
initialization of the filter and ensure the termination of the initialization phase by monitoring the internal TDOA error.

We experimentally evaluated the robustness and the accuracy of our approach with an ultrasound TDOA system in a wide indoor environment. For that, we constructed an ultrasound signal beacon and several integrated receiver units with WiFi communication capabilities and low powerconsumption. In extensive experiments we randomly navigated the signal beacon mounted on a R/C model car through the environment. Compared to a high-precision optical motion capture system our TDOA position estimates had an average localization error below $5 \mathrm{~cm}$, which slightly outperforms a state-of-the-art iterative optimization approach. Furthermore, we demonstrate the robustness of our filter in model car trajectories, where the beacon temporarily left the range of the receivers, and with a pedestrian carrying the beacon and partially occluding the line of sight between the beacon and the receivers. With additional randomly sampled disproportional measurement errors we demonstrate that our approach is robust to measurement outliers and outperforms a standard iterative optimization approach.

By not only estimating the position of a signal beacon, but extending the state estimation to the position of receivers, our localization system can operate without any prior positional information. With that self-calibration, the localization system is easy to set up, where the receivers are just distributed in a room and any target can be tracked immediately with high precision. Applications of our approach using the ultrasound system include localization in industrial logistics, e.g., autonomous transporters, or recreational activities where the relatively low-cost system could be quickly installed for a positional reference. Our approach might also be used for localization of mobile phones in large indoor environments, such as airports, trade fair halls, or supermarkets. The smart phones may, for example, emit a high-pitched sound, which is inaudible by human ears, and which is received by specific microphones to locate and guide the user.

This paper is organized as follows. After discussing related work in the following section, we state the problem definition in Section III and describe our approach to particle filter localization in Section IV. In Section V, we demonstrate the accuracy and robustness of our filter and compare it to alternative approaches.

\section{RELATED WORK}

Many popular approaches for indoor localization are based on the received strength of radio signals. They evaluate the Received Signal Strengh Indication (RSSI) of stationary satellites, e.g., WiFi access points, to estimate distances [1], [2] or they use fingerprinting [3] of the signal characteristics. While robust, these approaches often impose considerable effort in the installation and calibration, which is measuring the position of the satellites and learning the signal coverage. The accuracy of these systems ranges in the region of decimeters, decreasing rapidly in areas with low coverage of satellites. Additionally, in many cases the systems react sensitively to changes in the environment.

A promising alternative are systems based on the runtime of signals. Time of arrival (TOA) based approaches estimate the distance between peers by exchanging a signal and recording its send and receive times on time-synchronized devices. Alternatively, they use a transponder to bounce a signal and record its round trip delay at the origin [4], or emit two signals of different velocities, e.g., RF and ultrasound signals and compare the runtime differences [5], [6]. Once the distances between peers are known, the problem is reduced to the intersection of circles or spheres, which is a well-researched topic. Solutions are found using linear estimators [7], convex optimization [8], or iterative approaches [9], [10]. These TOA systems have in common, that all devices have to be precisely time-synchronized and usually the hardware delays of both sending and receiving a signal have to be calibrated in advance. Some other approaches use intermediate nodes to eliminate the hardware delays [11], which increases infrastructure complexity and costs.

We focus on systems based on TDOA, evaluating the hyperbolic relation of the runtime difference of a signal received by two receivers. In popular approaches the problem of locating and/or tracking a signal beacon is considered, using linear estimators, iterative approaches with squared or maximum likelihood estimators [12], particle filters [13], [14], [15] or Kalman filters [16], [17]. In all of these approaches the receiver positions are known in advance, which requires the calibration of their positions.

In recent years algorithms have evolved to localize such a signal beacon using TDOA without knowing the position of receivers at all. Some rely on an approximation of the signal propagation, e.g., that the path of the signal is a straight line [18], or that signals are emitted far away. In this far field case the distance vectors between receivers can be approximated [19], [20], or even calculated in closed form [21], [22]. Once the receivers are known, the calculation of the signal direction is straight forward.

For the general problem of TDOA, however, no analytical solutions for the mathematical constraints of the problem seem to exist [23], [24]. An elegant approach to general TDOA was presented by Pollefeys and Nister [24] using matrix factorization of the equation system. However, this approach can be only applied when at least eight (respectively ten in the three dimensional case) receivers are available which is twice the minimum number [25]. Furthermore, this technique appears to be prone to TDOA measurement errors. In contrast, root mean squares and maximum likelihood estimators behave more robust to Gaussian measurement errors. Biswas and Thrun address the problem using iterative gradient descent optimization [26]. These approaches require proper a initialization and tend to get stuck in local minima of the error function. Multiple repeated attempts with random initialization were proven to solve almost all simulated random scenarios [25], but the efficient initialization of a scenario remains an open problem. Besides, the iterative approaches, while robust to 
Gaussian errors, are still prone to disproportional errors, caused by echoes from walls, and by signal detection errors in the receivers.

Unlike the above-mentioned approaches, our algorithm can be reliably and efficiently initialized without any prior information and is very robust to Gaussian noise and disproportional errors in the TDOA measurements.

\section{Problem Formulation}

We consider the problem of simultaneous estimation of a continuous trajectory $\mathbf{S}_{t}(t=1, \ldots, m)$ of a signal beacon and the stationary positions $\mathbf{M}_{i}(i=1, \ldots, n)$ of $n$ receivers in Euclidean space $\mathbb{R}^{p}$, where $p \in\{2,3\}$. At each discrete time step $t$, the beacon emits a discrete signal at the unknown send time $s_{t}$. The signal is received and uniquely identified by some or all of the receivers at the times $r_{i t}$. For estimating the beacon trajectory and the receiver positions only the arrival times $r_{i t}$ of the signal are available. Up to time $t$ the measurement data $\mathbf{z}_{1: t}=\left\{\mathbf{z}_{j} \mid j=1, \ldots, t\right\}$ is available. As the range of the sound signal is limited and its propagation may be inhibited by obstacles, at each time step the measurement data $\mathbf{z}_{j} \subseteq\left\{r_{i j} \mid i=1, \ldots, n\right\}$ is a subset of the possible reception times of all receivers.

We assume that the signal propagates from the beacon to the receivers in a straight line with a constant signal velocity $c$ according to the signal propagation equation

$$
\left\|\mathbf{M}_{i}-\mathbf{S}_{t}\right\|=c\left(r_{i t}-s_{t}\right),
$$

where $\|\cdot\|$ is the Euclidean norm.

In the general TDOA localization problem [23], [25] the positions of signal beacons $\mathbf{S}_{j}$ and receivers $\mathbf{M}_{i}$ are arbitrary in space. As no anchor positions are provided, the position and the orientation of the signal beacon and the receivers relative to global coordinates is undetermined. The estimated solution can be compared to references by aligning it using a congruent transformation.

\section{Robust State Estimation in the Particle Filter}

We consider the estimation of the continuous trajectory of the signal beacon and the positions of the stationary receivers as a recursive state estimation problem. For that, we apply the Bayesian filtering scheme [27], which is a probabilistic approach to recursive state estimation. It is suitable to TDOA localization as the state can be computed online and it is robust to motion and measurement uncertainty. The key idea of the recursive Bayesian filter is to maintain a probability density $p\left(\mathbf{x}_{t} \mid \mathbf{z}_{1: t}, \mathbf{u}_{1: t}\right)$ of the state $\mathbf{x}_{t}$ at time $t$. The state is conditioned on all sensor data $\mathbf{z}_{1: t}$ and control commands $\mathbf{u}_{1: t}$ up to that time $t$. This probability density which is also called posterior distribution can be factorized into

$$
\begin{aligned}
& p\left(\mathbf{x}_{t} \mid \mathbf{z}_{1: t}, \mathbf{u}_{1: t}\right)=\eta_{t} p\left(\mathbf{z}_{t} \mid \mathbf{x}_{t}\right) \\
& \quad \int p\left(\mathbf{x}_{t} \mid \mathbf{u}_{t}, \mathbf{x}_{t-1}\right) p\left(\mathbf{x}_{t-1} \mid \mathbf{u}_{1: t-1}, \mathbf{z}_{1: t-1}\right) \mathrm{d} \mathbf{x}_{t-1},
\end{aligned}
$$

where $\eta_{t}$ is a normalizing constant ensuring that $\int p\left(\mathbf{x}_{t} \mid \mathbf{z}_{1: t}, \mathbf{u}_{1: t}\right) \mathrm{d} \mathbf{x}_{t}=1$. In (2), the term $p\left(\mathbf{x}_{t} \mid \mathbf{u}_{t}, \mathbf{x}_{t-1}\right)$ is the state transition probability and $p\left(\mathbf{z}_{t} \mid \mathbf{x}_{t}\right)$ is the measurement probability specified by the motion model and the sensor model, respectively.

Various implementations of the recursive Bayesian filter exist. The Kalman filter assumes Gaussian uncertainty and linear system dynamics. There are various extensions for nonlinear systems [27]. However, their performance degrades with increasing nonlinearities [28], and the Gaussian uncertainty assumption is not valid on TDOA data with additional measurement outliers.

We apply the particle filter, also known as Monte Carlo localization [29], which approximates the current belief $p\left(\mathbf{x}_{t} \mid \mathbf{z}_{1: t}, \mathbf{u}_{1: t}\right)$ by a set $\mathcal{M}=\left\{\left(\mathbf{x}^{[k]}, w^{[k]}\right) \mid k=1, \ldots, N\right\}$ of $N$ particles. Each particle corresponds to a state hypothesis $\mathbf{x}^{[k]}$ weighted by the so-called importance weight $w^{[k]}$. In the particle filter, we perform the recursive belief update given in (2) according to the following three steps:

1) In the prediction step, we propagate each particle by drawing a successor state based on the proposal distribution $p\left(\mathbf{x}_{t}^{[k]} \mid \mathbf{u}_{t}, \mathbf{x}_{t-1}^{[k]}\right)$ specified by the motion model. Thereby, the control command $\mathbf{u}_{t}$ and the motion uncertainty is taken into account.

2) In the correction step, we integrate a new measurement $\mathbf{z}_{t}$ by updating the weight $w_{t}^{[k]} \propto w_{t-1}^{[k]} p\left(\mathbf{z}_{t} \mid \mathbf{x}_{t}^{[k]}\right)$ of each particle according to the measurement likelihood given its state hypothesis. The measurement likelihood is defined in the sensor model taking into account the measurement process and the sensor characteristics.

3) In the resampling step, we draw a new set of particles from $\mathcal{M}$ with replacement such that each particle in $\mathcal{M}$ is selected with a probability that is proportional to its weight.

In particular, we reduce the particle depletion by applying low variance resampling [27] and omit the resampling step until the effective number of particles [30] $N_{\text {eff }}=\left(\sum_{k=1}^{N}\left(w^{[k]}\right)^{2}\right)^{-1}$ drops below half the number of particles.

The initialization of the filter and the design of the models described above is substantial for precise and reliable state estimation. In the following Sections IV-B to IV-D we describe our approach to robust initialization of the particle filter, a constant velocity motion model suitable for standard beacon tracking, and the TDOA sensor model.

For tracking of a mobile beacon, we define the state $\mathbf{x}_{t}$ at time $t$ as

$$
\mathbf{x}_{t}=\left[\mathbf{S}_{t}^{T}, \mathbf{V}_{t}^{T}, \mathbf{M}_{1 t}^{T}, \ldots, \mathbf{M}_{n t}^{T}\right]^{T} .
$$

Here, $\mathbf{S}_{t}$ is the position and $\mathbf{V}_{t}$ is the velocity of the beacon. Furthermore, we neglect the fact that the receiver positions $\mathbf{M}_{i}$ are assumed to be stationary, which will be taken into account in the motion model as described in Section IV-C.

\section{A. Calculation of the Particle Filter Estimate}

In this paper, we estimate both, the position of the signal beacon, as well as the positions of the receivers. For calculation of the filter estimate from the weighted set of particles we approximate the posterior distribution $p\left(\mathbf{x}_{t} \mid \mathbf{z}_{1: t}, \mathbf{u}_{1: t}\right)$ by a 
Gaussian. According to that approximation, the filter estimate $\hat{\mathbf{x}}_{t}$ is the weighted average of the particles which is the mean of the Gaussian distribution.

In our state estimation problem the TDOA measurement data specifies only the individual distances between the receivers and the signal origins, i.e., the internal configuration of the system. Consequently, the global translation and rotation of the state $\mathbf{x}^{[k]}$ of every particle in the filter is arbitrary. To evaluate the weighted average of all particles we globally align the particles in a congruent transformation. The constraints inside the configuration estimated by every particle, and the distances between the beacon and the receivers remain unchanged in this procedure.

We identify the transformation parameters between two particle states $\mathbf{x}^{[k]}$ and $\mathbf{x}^{[l]}$, the rotation matrix $R$ and the translation vector $\tau$, by minimizing the mean squared error

$$
e^{2}(R, \boldsymbol{\tau})=\frac{1}{n} \sum_{i=1}^{n}\left\|\mathbf{M}_{i}^{[k]}-\left(R \mathbf{M}_{i}^{[l]}+\boldsymbol{\tau}\right)\right\|^{2}
$$

of the receiver positions, where $\mathbf{M}_{i}^{[k]}$ is the position of the $i$-th receiver in the $k$-th particle. For that we follow the approach of Arun et al. [31] and apply singular value decomposition to determine the rotation matrix. In the first step, we calculate the mean

$$
\boldsymbol{\mu}^{[k]}=\frac{1}{n} \sum_{i=1}^{n} \mathbf{M}_{i}^{[k]} \quad \text { and } \quad \boldsymbol{\mu}^{[l]}=\frac{1}{n} \sum_{i=1}^{n} \mathbf{M}_{i}^{[l]}
$$

of the receiver positions of both particles, so that the translation can be expressed as $\boldsymbol{\tau}_{k l}=\boldsymbol{\mu}^{[k]}-\boldsymbol{\mu}^{[l]}$. The joint covariance of $\mathbf{M}^{[k]}$ and $\mathbf{M}^{[l]}$ can be estimated as

$$
\Sigma_{k l}=\frac{1}{n} \sum_{i=1}^{n}\left(\mathbf{M}_{i}^{[k]}-\boldsymbol{\mu}^{[k]}\right)\left(\mathbf{M}_{i}^{[l]}-\boldsymbol{\mu}^{[l]}\right)^{T} .
$$

Then the optimal rotation matrix with respect to (4) is $R_{k l}=U V^{T}$, where $U D V^{T}$ is the singular value decomposition of $\Sigma_{k l}$. As the configuration of the receivers can be mirrorinverted, the determinant of this rotation is $\operatorname{det}\left(R_{k l}\right)= \pm 1$.

We align all particles to the most likely particle, which we assume to be the first particle in the set. The alignment of every receiver and the signal beacon in a particle is calculated by

$$
\begin{aligned}
\tilde{\mathbf{M}}_{i}^{[k]} & =R_{1 k}\left(\mathbf{M}_{i}^{[k]}-\boldsymbol{\mu}^{[k]}\right)+\boldsymbol{\mu}^{[1]} \\
\tilde{\mathbf{S}}^{[k]} & =R_{1 k}\left(\mathbf{S}^{[k]}-\boldsymbol{\mu}^{[k]}\right)+\boldsymbol{\mu}^{[1]}
\end{aligned}
$$

from the rotation matrix and the mean of each estimate of the receiver positions. Analogously, the velocity estimate is transformed by $\tilde{\mathbf{V}}^{[k]}=R_{1 k} \mathbf{V}^{[k]}$.
Now, the weighted mean and the covariance of the particle filter with normalized weights can be calculated by

$$
\begin{aligned}
\boldsymbol{\mu}_{t} & =\sum_{k=1}^{N} w^{[k]} \tilde{\mathbf{x}}_{t}^{[k]} \\
\Sigma_{t} & =\sum_{k=1}^{N} w^{[k]}\left(\tilde{\mathbf{x}}_{t}^{[k]}-\boldsymbol{\mu}_{t}\right)\left(\tilde{\mathbf{x}}_{t}^{[k]}-\boldsymbol{\mu}_{t}\right)^{T} .
\end{aligned}
$$

\section{B. Initialization}

As long as the particle filter is in the initialization stage, we try to initialize the filter using an iterative non-probabilistic error minimization approach. In this stage, we periodically initialize a certain proportion of the particles according to the result of an iterative minimization on a subset of TDOA measurement data as follows. In an initialization attempt at time step $t$, we use a gradient descent approach, followed by Newton's method [25] to optimize the signal propagation constraint (1). For that, we randomly choose $\tilde{m}<t$ beacon signals including the latest signal $\left\{\mathbf{z}_{I_{1}}, \ldots, \mathbf{z}_{I_{\tilde{m}-1}}, \mathbf{z}_{t}\right\} \subseteq \mathbf{z}_{1: t}$ as a subset of the available measurement data, where $I_{j}(j=1, \ldots, \tilde{m}-1)$ is the random index of the subset. For simplicity in notation, in the following we use $j$ to denote the measurement with the index $I_{j}$.

The signal propagation constraint (1) yields a maximum of $\tilde{m} n$ equations if all receivers obtain all signals, with $(p+1) \tilde{m}+p n$ unknown variables. By combining two equations for receivers $i$ and $k(1 \leq i, k \leq n)$ and for a signal $j$ the signal emission time $s_{j}$ is eliminated, reducing the maximum number of equations to $\tilde{m}(n-1)$ equations and $p \tilde{m}+p n$ variables. We obtain the hyperbolic equation

$$
\left\|\mathbf{M}_{i}-\mathbf{S}_{j}\right\|-\left\|\mathbf{M}_{k}-\mathbf{S}_{j}\right\|=c\left(r_{i j}-r_{k j}\right) .
$$

We define the state vector with signal and receiver positions to optimize as

$$
\mathbf{v}=\left[\tilde{\mathbf{S}}_{1}^{T}, \ldots, \tilde{\mathbf{S}}_{\tilde{m}}^{T}, \tilde{\mathbf{M}}_{1}^{T}, \ldots, \tilde{\mathbf{M}}_{n}^{T}\right]^{T} .
$$

Without loss of generality, we choose $k=1$ and define the hyperbolic error function

$$
\mathbf{b}(\mathbf{v})=\left[f_{21}(\mathbf{v}), f_{22}(\mathbf{v}), \ldots, f_{n \tilde{m}}(\mathbf{v})\right]^{T}
$$

with the error functional

$$
f_{i j}(\mathbf{v})=\left\|\tilde{\mathbf{M}}_{i}-\tilde{\mathbf{S}}_{j}\right\|-\left\|\tilde{\mathbf{M}}_{k}-\tilde{\mathbf{S}}_{j}\right\|-c\left(r_{i j}-r_{k j}\right) .
$$

We search for the optimal estimate of $\mathbf{v}$ by minimizing the squared norm of the error function

$$
\hat{\mathbf{v}}=\underset{\mathbf{v}}{\operatorname{argmin}} \frac{1}{2} \mathbf{b}(\mathbf{v})^{T} \mathbf{b}(\mathbf{v}) .
$$

The iterative search is initialized by a random estimate $\mathbf{v}^{(0)}$ close to zero. As we have no anchor points or prior information, this is a reasonable guess.

In every iteration step $h$ we calculate the first term of the Taylor series at the current estimate $\mathbf{v}^{(h)}$, the Jacobian

$$
Q=\frac{\partial \mathbf{b}}{\partial \mathbf{v}} \text {. }
$$


We apply a gradient descent optimization to minimize the objective $w=\frac{1}{2} \mathbf{b}^{T} \mathbf{b}$ at the position $\mathbf{v}$. The update vector $\Delta \mathbf{v}$ is then calculated by the direction of steepest descent

$$
\Delta \mathbf{v}=\gamma^{(h)} \nabla w=\gamma^{(h)} Q^{T} \mathbf{b}
$$

with an adaptive step width $\gamma^{(h)}$. The next estimate is calculated by $\mathbf{v}^{(h+1)}=\mathbf{v}^{(h)}-\Delta \mathbf{v}$.

When the gradient descent algorithm has converged such that $\|\Delta \mathbf{v}\|<\epsilon_{1}$ we switch to the faster Newton algorithm. Here, in every intersection we use the least squares method to calculate the zero of the first Taylor series term, which results in the update vector $\Delta \mathbf{v}$ :

$$
\left(Q^{T} Q\right) \Delta \mathbf{v}=Q^{T} \mathbf{b} \quad \Rightarrow \quad \Delta \mathbf{v}=\left(Q^{T} Q\right)\left(Q^{T} \mathbf{b}\right) .
$$

Again, we calculate the next estimate $\mathbf{v}^{(h+1)}$ by subtracting the update vector. We terminate when $\|\Delta \mathbf{v}\|<\epsilon_{2}$.

The sequence of gradient descent steps, followed by Newton's method is an effort to improve the convergence of the algorithm, as the unpredictability of Newton's method on the error function denies its application from the beginning.

The result of the iterative approach describes an approximation of the latest position of the beacon $\mathbf{S}_{t}$ and the stationary positions of the receivers $\mathbf{M}_{1}$ to $\mathbf{M}_{n}$ :

$$
\tilde{\mathbf{x}}=\left[\tilde{\mathbf{S}}_{t}^{T}, \mathbf{0}^{T}, \tilde{\mathbf{M}}_{1}^{T}, \ldots, \tilde{\mathbf{M}}_{n}^{T}\right]^{T} .
$$

We inject this approximation as a possible initialization into the particle filter by sampling a certain proportion of the particle set from the multivariate Gaussian distribution $\mathcal{N}\left(\tilde{\mathbf{x}}, \Sigma_{\text {Newton }}\right)$. Here, the covariance $\Sigma_{\text {Newton }}$ of the Newton optimization results with respect to the true state can be determined straight forward from recorded data.

Some of these attempts of the iterative algorithm may not be successful. In fact, especially in the beginning, when only few signals are given and the signal beacon did not move that far so that the positions of the signal emissions are not well distributed, most optimization attempts will fail or converge to a local minimum. However, the probability of finding the correct solution is increased by repeated attempts [25]. Furthermore, by using varying subsets of data, we circumvent adverse scenarios, where the iterative algorithm never finds a solution.

After some time, the initialization algorithm has provided an estimate which is close to the true configuration and the particle filter converges towards the correct positions. We detect this by continuously evaluating the internal TDOA error $\left\|\left[f_{1}, \ldots, f_{n}\right]\right\|$ of the particle filter mean $\boldsymbol{\mu}_{t}$, where

$$
f_{i}\left(\boldsymbol{\mu}_{t}\right)=\left\|\tilde{\mathbf{M}}_{1 t}-\tilde{\mathbf{S}}\right\|-\left\|\tilde{\mathbf{M}}_{i t}-\tilde{\mathbf{S}}\right\|-c\left(r_{1 t}-r_{i t}\right) .
$$

After a possible initialization was found, a lowpass filtered indicator of the TDOA error will fall below a threshold $\delta_{\text {init }}$, which is proportional to the noise of the system. Empirically, we have determined this value to $\delta_{\text {init }}=1$, which is suitable for all experiments that we conducted, reliably indicating proper convergence.

Naturally, the internal TDOA error of the position estimate is not a safe guarantee that a possible solution of low TDOA error is also the correct solution with low deviation from the reference positions. The discrepancy becomes evident when additional measurements are received which are not compatible to the current state, so the TDOA error will then increase to a high level. We allow the algorithm to re-enable initialization mode if such an inconsistency occurs, which is triggered by the internal error rising above a threshold of $\delta_{\text {retry }}=2$.

\section{Motion Model}

The proper design of the probabilistic motion model is substantial for the efficiency and the accuracy of the state estimation during the prediction step of the particle filter. In the context of mobile beacon tracking with simultaneous calibration of the receiver positions we factorize the probabilistic motion model into

$$
\begin{array}{r}
p\left(\mathbf{x}_{t}^{[k]} \mid \mathbf{u}_{t}, \mathbf{x}_{t-1}^{[k]}\right)=p\left(\mathbf{S}_{t}^{[k]}, \mathbf{V}_{t}^{[k]} \mid \mathbf{u}_{t}, \mathbf{S}_{t-1}^{[k]}, \mathbf{V}_{t-1}^{[k]}\right) \\
\cdot \prod_{j=1}^{n} p\left(\mathbf{M}_{j t}^{[k]} \mid \mathbf{u}_{t}, \mathbf{M}_{j t-1}^{[k]}\right)
\end{array}
$$

assuming an independent motion of the beacon and the individual receivers.

In most applications no controls of the movement of the beacon are given. Hence, we assume the control command $\mathbf{u}_{t}=\Delta t$ to contain only the time elapsed since the last prediction step and apply a constant velocity motion model. This model in principle assumes that the beacon moves with a constant velocity and models changes in the velocity by a covariance matrix $\Sigma_{\mathbf{V}}$, which can be determined from empirical data. According to these assumptions the probabilistic model is

$$
\begin{aligned}
\mathbf{S}_{t+1} & =\mathbf{S}_{t}+\mathbf{V}_{t} \Delta t \\
\mathbf{V}_{t+1} & =\mathbf{V}_{t}+\boldsymbol{\zeta}_{t} \quad \text { with } \quad \boldsymbol{\zeta}_{t} \sim \mathcal{N}\left(\boldsymbol{\zeta}_{t} ; \mathbf{0}, \Sigma_{\mathbf{V}}\right) .
\end{aligned}
$$

The positions of the stationary receivers do not have to be modified so that the probability density function of the motion model

$$
p\left(\mathbf{M}_{j t}^{[k]} \mid \mathbf{u}_{t}, \mathbf{M}_{j t-1}^{[k]}\right)=\delta\left(\mathbf{M}_{j t-1}^{[k]}\right)
$$

of the receivers is the Dirac delta distribution. This results in a sample degeneracy, or "attrition", which is usually prevented by adding small random Gaussian disturbances to the receiver positions of each sample during resampling. However, this leads to a loss of information in each resampling step, and hence is a suboptimal solution. We use kernel smoothing [32] instead, as soon as the particle filter is converged:

$$
\mathbf{M}_{j t}^{[k]} \sim \mathcal{N}\left(\mathbf{M}_{j t}^{[k]} ; a \mathbf{M}_{j t-1}^{[k]}+(1-a) \overline{\mathbf{M}}_{j t-1}, h^{2} \Sigma_{M_{j t-1}}\right) .
$$

$\overline{\mathbf{M}}_{j t-1}$ and $\Sigma_{M_{j t-1}}$ are the weighted mean and covariance of the $j$-th receiver position over the particle set at time $t-1$. The constant factors $a=\frac{3 \gamma-1}{2 \gamma}$ and $h^{2}=1-a^{2}$ only depend on a discount factor $\gamma$, which we set to 0.95 . Compared to adding disturbances, kernel smoothing prevents a loss of information in the receiver positions. Before each kernel smoothing step the particle set is aligned as described in Section IV-A. 


\section{Sensor Model}

The probabilistic sensor model $p(\mathbf{z} \mid \mathbf{x})$ defines the likelihood of the measurement data $\mathbf{z}$ given the state $\mathbf{x}$ of the system. Here, the measurement data $\mathbf{z}_{t}=\left\{z_{1}, \ldots, z_{k}\right\} \subseteq$ $\left\{r_{i t} \mid i=1, \ldots, n\right\}$ is a subset of all possible reception times of the beacon signal emitted at time $s_{t}$ measured by the receivers. We assume the measurements of the individual receivers to be independent given the current state $\mathrm{x}$ of the system, i.e., the position of the beacon and the receivers:

$$
p(\mathbf{z} \mid \mathbf{x})=\prod_{j=1}^{k} p\left(z_{j} \mid \mathbf{x}\right) .
$$

Based on Eq. (1) the measurement process of each individual measurement $z_{j}$ is described by

$$
z_{j}-s_{t}=\frac{1}{c}\left\|\mathbf{M}_{j}-\mathbf{S}_{t}\right\|+\varepsilon_{j} .
$$

In our model, we assume that the corresponding error variable $\varepsilon_{j}$ is basically Gaussian distributed with an additional small proportion of extreme outliers induced by external influences like reflections of the signal and echoes. Consequently, we apply a mixed model combining a Gaussian and a uniform distribution:

$$
p\left(\varepsilon_{j}\right)=\alpha \frac{1}{2 \delta}+(1-\alpha) \mathcal{N}\left(\varepsilon_{j} ; 0, \sigma^{2}\right) .
$$

Here, $\delta$ is the maximum error of measurement outliers and we define the proportion of measurement outliers by the parameter $0 \leq \alpha \leq 1$.

Based on this measurement error, the measurement likelihood is defined as

$$
p\left(z_{j} \mid \mathbf{x}\right)=\alpha \frac{1}{2 \delta}+(1-\alpha) \mathcal{N}\left(z_{j} ; \mu_{j}, \sigma^{2}\right)
$$

with the mean $\mu_{j}=s_{t}+\frac{1}{c}\left\|\mathbf{M}_{j}-\mathbf{S}_{t}\right\|$.

To maximize the likelihood of the measured data with respect to the expected measurements given the current pose of the beacon, we estimate the most likely send time $\hat{s}_{t}$ :

$$
\hat{s_{t}}=\underset{\tilde{s}_{t}}{\operatorname{argmax}} p\left(\mathbf{z} \mid \mathbf{x}, \tilde{s}_{t}\right)=\underset{\tilde{s}_{t}}{\operatorname{argmax}} \prod_{j=1}^{k} p\left(z_{j} \mid \mathbf{x}, \tilde{s}_{t}\right) \text {. }
$$

Maximizing Eq. (30) requires to solve

$$
\begin{aligned}
& \frac{\mathrm{d}}{\mathrm{d} \hat{s}_{t}} p\left(\mathbf{z} \mid \mathbf{x}, \hat{s}_{t}\right) \\
& =\frac{\mathrm{d}}{\mathrm{d} \hat{s}_{t}} \prod_{j=1}^{k} p\left(z_{j} \mid \mathbf{x}, \hat{s}_{t}\right) \\
& =\frac{\mathrm{d}}{\mathrm{d} \hat{s}_{t}} \prod_{j=1}^{k}\left(\frac{\alpha}{2 \delta}+(1-\alpha) \mathcal{N}\left(z_{j} ; \hat{s}_{t}+\frac{\left\|\mathbf{M}_{j}-\mathbf{S}_{t}\right\|}{c}, \sigma^{2}\right)\right) \\
& \stackrel{!}{=} 0 .
\end{aligned}
$$

This product results in a binomial term of degree $k$, depending on the number of available receiver measurements. For that derivative, we see no way to find a direct solution in a closed form. A practical solution can be found using iterative optimization with only a few iterations. However, as the likelihood maximization is calculated in every observation step and for every particle in the filter this will eventually be very slow.

Hence, we approximate the most likely send time by neglecting the (comparably small) uniform part of the measurement likelihood and obtain

$$
\begin{aligned}
0= & \frac{\mathrm{d}}{\mathrm{d} \hat{s}_{t}} \prod_{j=1}^{k} \mathcal{N}\left(z_{j} ; \hat{s}_{t}+\frac{\left\|\mathbf{M}_{j}-\mathbf{S}_{t}\right\|}{c}, \sigma^{2}\right) \\
= & \left(\frac{1}{\sqrt{2 \pi} \sigma}\right)^{k} \exp \left(-\frac{\sum_{j=1}^{k}\left(z_{j}-\hat{s}_{t}-\frac{\left\|\mathbf{M}_{j}-\mathbf{S}_{t}\right\|}{c}\right)^{2}}{2 \sigma^{2}}\right) \\
& \frac{1}{\sigma^{2}} \sum_{j=1}^{k}\left(z_{j}-\hat{s}_{t}-\frac{\left\|\mathbf{M}_{j}-\mathbf{S}_{t}\right\|}{c}\right) .
\end{aligned}
$$

As the first and the second factor are always greater than zero, the equation can be rewritten as

$$
0=\frac{1}{\sigma^{2}} \sum_{j=1}^{k}\left(z_{j}-\hat{s}_{t}-\frac{\left\|\mathbf{M}_{j}-\mathbf{S}_{t}\right\|}{c}\right),
$$

which finally can be solved to

$$
\hat{s}_{t}=\frac{1}{k} \sum_{j=1}^{k}\left(z_{j}-\frac{\left\|\mathbf{M}_{j}-\mathbf{S}_{t}\right\|}{c}\right) .
$$

With that approximation of $\hat{s}_{t}$, we calculate the likelihood of a measurement $\mathbf{z}_{t}$ in (26) with

$$
p\left(z_{j} \mid \mathbf{x}\right)=\alpha \frac{1}{2 \delta}+(1-\alpha) \mathcal{N}\left(z_{j} ; \hat{s}_{t}+\frac{1}{c}\left\|\mathbf{M}_{j}-\mathbf{S}_{t}\right\|, \sigma^{2}\right)
$$

taking into account measurement outliers for robust beacon tracking in the particle filter.

\section{EXPERIMENTS}

We have verified the capability of our approach in extensive experiments in an indoor test setting. In our experiments we use a two-dimensional setting of receivers in an oval arrangement and a signal beacon to be tracked, similar to the testbed in the experiment of tracking a moving model train [25].

\section{A. Experimental Setup}

For the experiments we use an ultrasound system, which consists of a moving beacon and multiple stationary receiver devices. The beacon (see Fig. 2) emits short bursts at $40 \mathrm{kHz}$ frequency, which can be detected by the receivers. It does not communicate with the receivers, so that it fits into a small case and can operate battery powered. Our prototypical ultrasound beacon has a diameter of $6.4 \mathrm{~cm}$ and weighs 44 grams, so that it can be attached to moving objects or can be carried by hand.

Each receiver device consists of an ultrasound capsule attached to a custom controller board amplifying and digitizing the signal. The controller board is connected to a computer which detects the ultrasound bursts of the beacon and calculates their points in time. Furthermore, for a flexible integration 


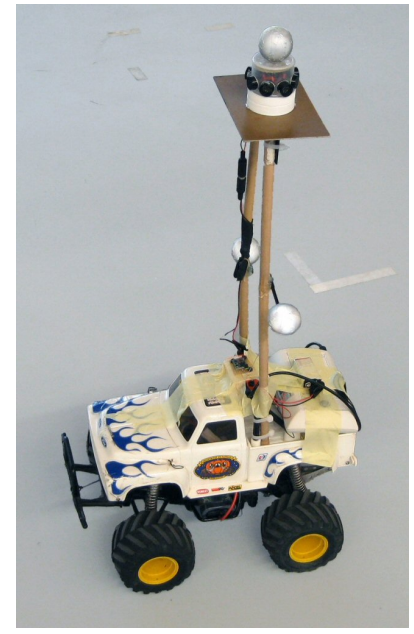

Fig. 2. The ultrasound beacon mounted onto the top of a R/C model car For ground truth information reflective MoCap markers are attached to the car and the beacon and allow for precise high-speed tracking of the position of the beacon.

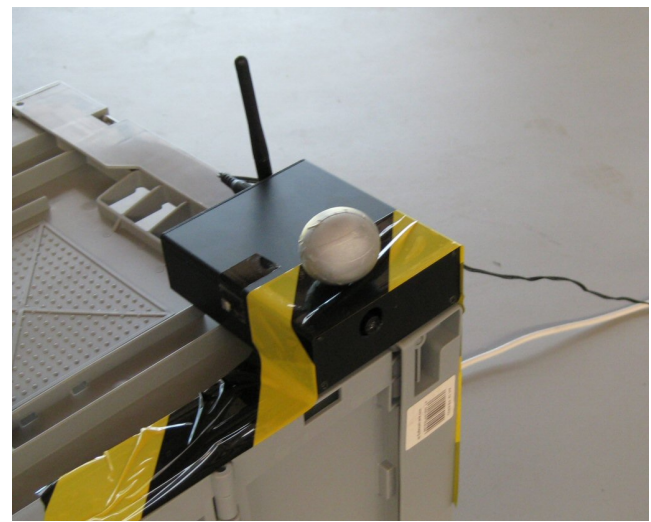

Fig. 3. One of our integrated receiver units with a Gumstix ${ }^{\circledR}$ Overo $^{\circledR}$ computer and wireless communication. A reflective MoCap marker is placed above the ultrasound microphone.

into arbitrary environments we have built integrated receiver units that do not require an external computer. These are based on Gumstix ${ }^{\circledR}$ Overo ${ }^{\circledR}$ Computer-on-Modules (COMs) with an ARM Cortex-A8 processor (see Fig. 3). With their low power consumption and the small dimensions of $12 \mathrm{~cm} \times 11 \mathrm{~cm} \times$ $4 \mathrm{~cm}$ they can be battery powered and quickly installed into the environment. For both versions we use a WiFi connection for data communication and time synchronization of the receivers. The latter is a critical factor for TDOA localization. In a $802.11 \mathrm{~g}$ network we achieve a precision of $0.1 \mathrm{~ms}$ by using a method based on message exchange between the peers [22].

Our present ultrasound system consists of five integrated receiver units and additional six conventional ultrasound receiver devices connected to laptop computers. The receivers were arranged in a roughly oval shaped formation on the same level (see Fig. 1) with the ultrasound microphone directed towards the center of the tracking area. As pointed out in Section III, our algorithm works in two- or three-dimensional space so that our implementation can be extended to three dimensions.
For continuous tracking we programmed the beacon to emit an ultrasound burst in a fixed interval of $300 \mathrm{~ms}$. Although our tracking system does not rely on fixed intervals, the $300 \mathrm{~ms}$ interval turned out to allow for a precise and all-over tracking of the moving beacon while the receivers can still uniquely identify the individual bursts.

To evaluate the precision and robustness of our ultrasound tracking system we use a MotionAnalysis optical motion capture system (MoCap) with nine Raptor-E cameras. The MoCap cameras were installed at the ceiling of the experimental area observing the full area inside the microphone array. The position of the microphones were obtained by attaching a reflective marker to each receiver exactly above the microphone. We attached another marker to the top of the ultrasound beacon, so that the trajectory of the sender could also be determined at a tracking frequency of $100 \mathrm{~Hz}$. In an experiment, where the beacon is carried by a person, he wore a hat with reflective markers on its top to obtain the ground-truth positions of the person instead of the position of the beacon.

For the first experiment, which we denote as the model car experiment, we installed the ultrasound beacon to an $\mathrm{R} / \mathrm{C}$ model car on a pole, such that it resides at the same height as the receivers (see Fig. 2). In several runs of three to ten minutes we navigated the model car through the experimental area, at varying velocities at an average of $1.5 \mathrm{~m} / \mathrm{s}$ and up to $4 \mathrm{~m} / \mathrm{s}$. In Exp. 1 the path of the model car included sharp turns, sudden stops, and rapid starts. In Exp. 2 we extended the movements by leaving the perimeter of the ultrasound microphones and the optical reference and re-entering at another location to force the particle filter to recover at totally different positions. Naturally, the signal is not received by all receivers, as the car navigates into a corner of the area, or even outside.

In the pedestrian experiment (Exp. 3) a person carried the sender in his hand. He walked a random path of a few minutes through the area, with sharp turns and stops. At all times, he held the beacon at the same height as the receivers to satisfy the planar constraint. In this experiment the direct path of the ultrasound signal to the receivers was obstructed by the body of the person, blocking some of the signals. Most notably, the ultrasound beacon and the hat with the reflective markers were not vertically aligned, such that their horizontal positions differed up to half a meter. Consequently, the reference positions are less reliable in this experiment.

We have split the experiments into several data sets to evaluate the behavior of the algorithm from different aspects. We obtain four data sets from the model car, namely Exp. 1a, Exp. $1 b$, Exp. $2 a$, and Exp. 2b. From the pedestrian experiment we obtain the data sets Exp. $3 a$ and Exp. $3 b$.

\section{B. Initialization Phase}

In the beginning, no positions of neither the beacon nor the receivers are known. The particle filter algorithm is initialized with a uniform distribution. As the number of measurements increases, the iterative TDOA algorithm described in Section IV-B begins to compute configurations of likely receiver positions and the beacon using a random subset of the available 


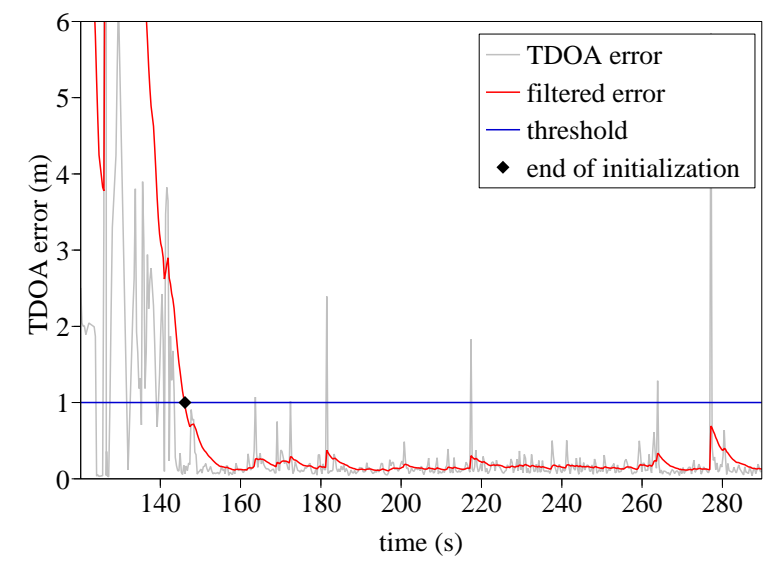

Fig. 4. TDOA error of Exp. 1a. After 26 seconds the filtered error falls below the limit of $\delta_{\text {init }}=1$. Then, initialization with the iterative algorithm ends. Note that the TDOA error is different from the position error of the beacon compared to the MoCap reference.

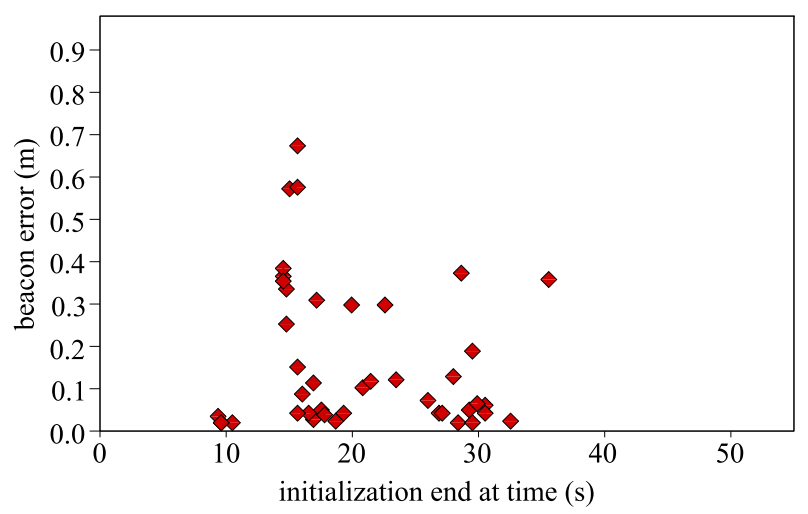

Fig. 5. The end time of the initialization (which is triggered by the TDOA error falling below $\delta_{\text {init }}=1$ ) and the corresponding localization error for six particle filter runs on each of the six data sets. In all runs the initialization time does not exceed 35 seconds.

signals. We choose to use the algorithm with a fixed number of 10 signals, which is a compromise between computational load and a good chance of converging.

We have evaluated the initialization scheme described in Section IV-B with six runs for each of the six data sets. During the initialization period the beacon moved on a curvy trajectory in the experimental area, which we require to initialize. Our localization algorithm monitors and low-pass filters the internal TDOA error of the particle filter estimate as shown in Fig. 4. It automatically finishes the initialization phase as soon as the filtered TDOA error drops below the threshold $\delta_{\text {init }}=1$. As shown in Fig. 5, the end of initialization of the filter is achieved in all of the tested scenarios after a maximum initialization time of 35 seconds. When the initialization was finished, the position error of the beacon was already below $70 \mathrm{~cm}$ in all cases, which indicates a proper initialization near to the true state.

\section{Tracking Phase}

After the initialization phase was finished, we deactivate the iterative algorithm and switch to position tracking in the
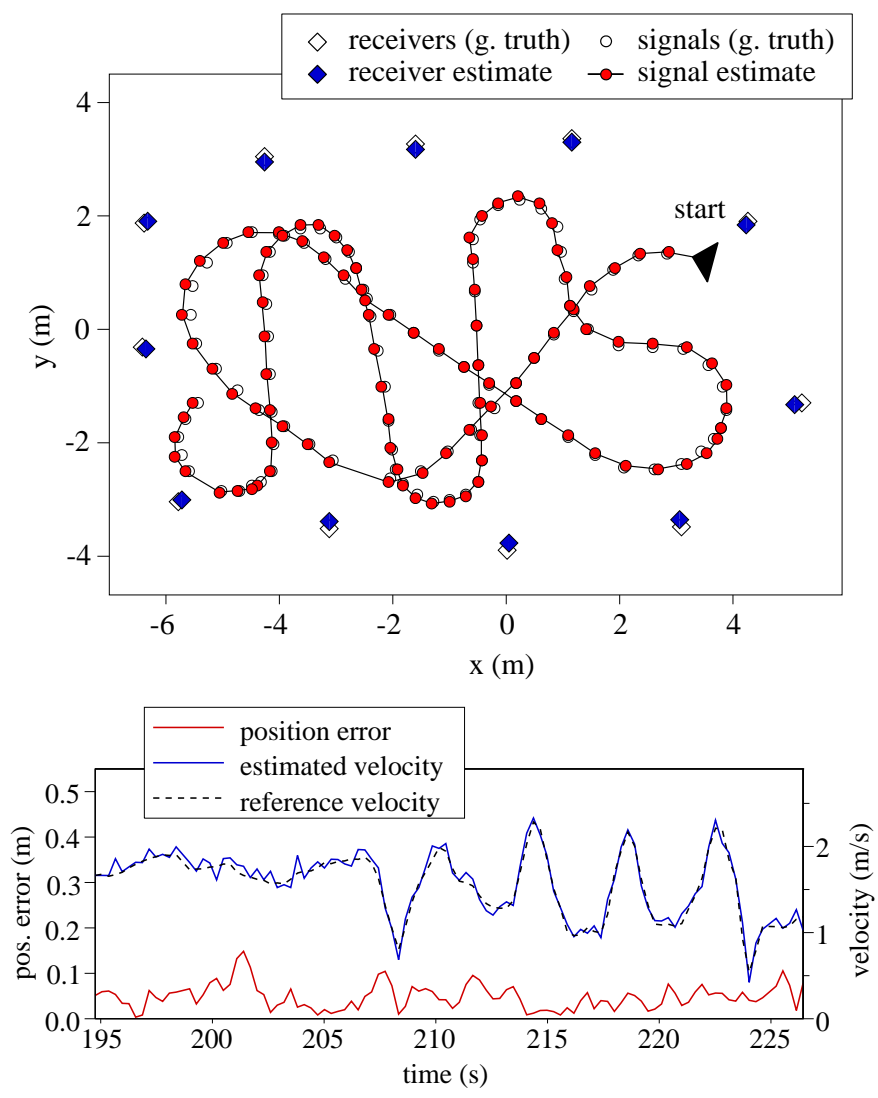

Fig. 6. An extract of 30 seconds of Exp. 1a. Top: In sharp corners the particles are slightly drawn to the outside - an effect of the constant velocity motion model. Bottom: Position error and velocity of the beacon. The mean position error is $4.8 \mathrm{~cm}(\sigma=2.8 \mathrm{~cm})$.

particle filter. Additionally, we enable kernel smoothing on the receiver estimates to prevent from information loss during the resampling step. Fig. 1 depicts an illustration of the beacon and the receiver estimate in the particle filter.

During the tracking phase the estimated beacon positions are very consistent to the indicated references. We observe an effect of the constant velocity motion model, which assumes a straight path of the beacon and consequently pushes the particles slightly to the outside curve. In an extract of 30 seconds of the first data set Exp. Ia we find that $95 \%$ of all positions deviate less than $10 \mathrm{~cm}$ from the reference positions, at a mean of $4.8 \mathrm{~cm}$ (Fig. 6).

In Exp. 2 we navigated the model car outside the range of the receivers, re-entering at another position after three to five seconds. As shown in Fig. 7, the estimate of the beacon diverges, as soon as not enough TDOA measurement data is available. However, a few steps after re-entering the range of the receivers new measurement data is received and the particle filter quickly recovers to the correct position.

\section{Robustness to Outliers and Comparison of Algorithms}

A common problem for TDOA localization in indoor environments are extreme measurement errors caused by echoes from nearby walls, noise from the environment, and wrong 


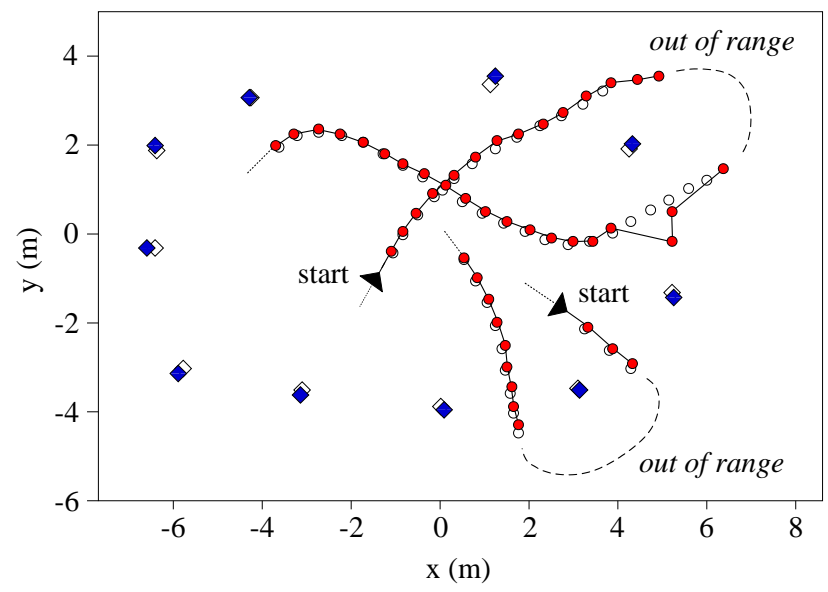

Fig. 7. Data set Exp. 2a: The beacon estimate when the signal is lost. Only a few steps after new TDOA information is available the particle filter estimate reliably converges to the true state.

\begin{tabular}{|c|c|}
\hline$\times \times \times>4.00 \mathrm{~m}$ & $\triangleright \backslash 0.13-0.25 \mathrm{~m}$ \\
\hline$\square 2.00-4.00 \mathrm{~m}$ & $0.07-0.13 \mathrm{~m}$ \\
\hline पायागय 1.00 - $2.00 \mathrm{~m}$ & 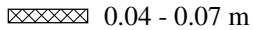 \\
\hline $0.50-1.00 \mathrm{~m}$ & $\times \times \times \quad 0.02-0.04 \mathrm{~m}$ \\
\hline$\measuredangle \quad 0.25-0.50 \mathrm{~m}$ & $\square<0.02 \mathrm{~m}$ \\
\hline
\end{tabular}
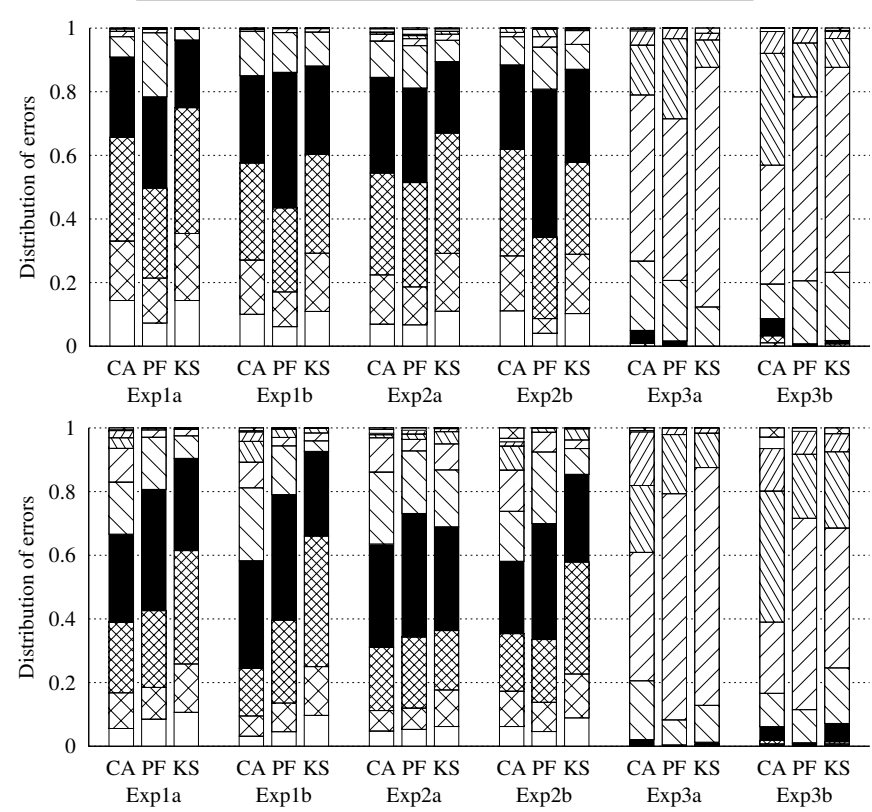

Fig. 8. The error distribution of the two model car experiments Exp. 1 and Exp. 2 and of the pedestrian experiment Exp. 3, comparing Cone Alignment (CA), particle filtering without (PF) and with (KS) kernel smoothing. Top: No outliers. Bottom: Simulated outliers with $100 \mathrm{~ms}$ standard deviation.

assignment of signals. These typical errors appear as single spikes in the TDOA graph, with magnitudes of $10 \mathrm{~ms}$ to more than $100 \mathrm{~ms}$. We verify the robustness of our particle filtering approach by a systematical simulation of such spikes.

We compared our algorithm to the Iterative Cone Alignment algorithm [25]. This iterative optimization approach minimizes the constraints between signals and receivers according to Eq. (1) in terms of energy minimization. For every new signal we call the algorithm with a set of 80 previous signals.

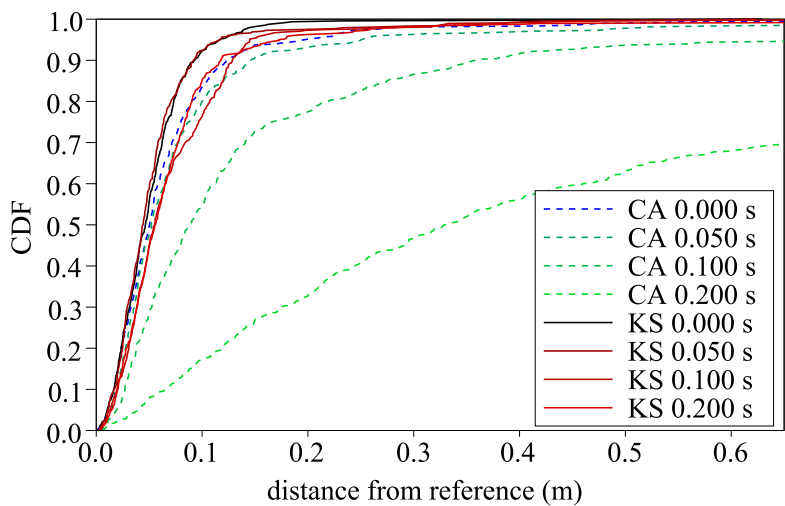

Fig. 9. The cumulative density of the position errors of the beacon for Cone Alignment (CA) and for the particle filter with kernel smoothing (KS) in data set Exp. 1a for varying magnitudes of measurement outliers. Whereas the particle filter is robust even to extreme outliers of $200 \mathrm{~ms}$, the Cone Alignment algorithm shows an increasing proportion of wrong estimates.

Although defective measurements are carried for a longer time, the high number of measurements taken into account increases the precision and the robustness of the algorithm.

For the evaluation of the robustness of the algorithms we systematically manipulated our measurement data by randomly generating outliers every 50 to 70 measurements. Their magnitudes were sampled from a Gaussian distribution with standard deviations of 10, 20, 50, 100, 200 and 500 milliseconds. For all of the six data sets we compare the deviation of the estimated positions from the reference positions for the different standard deviations.

Fig. 8 shows the error distribution of all algorithms with and without outliers. In all data sets without outliers the particle filter with kernel smoothing performs slightly better than the iterative approach. In contrast, in the presence of outliers, even the particle filter without kernel smoothing outperforms the Cone Alignment algorithm which is susceptible to these errors. Especially with kernel smoothing the particle filter performs robustly as soon as the estimates of the receiver positions are converged. The position errors in case of different magnitudes of outliers are illustrated in Fig. 9. As the estimates of the signal and receiver positions are related through the TDOA constraints between them, a wrongly estimated beacon position, caused by an outlier in the TDOA data, can also corrupt the receiver position estimates. In this way, the estimate of iterative algorithms like the Cone Alignment can literally "explode", resulting in a complete loss of the estimate.

\section{CONCLUSIONS AND FUture Work}

In this paper, we have presented a novel approach to robust localization of a mobile beacon using TDOA measurement data when no receiver positions are given. We cope with the high-dimensional state estimation problem, which typically has ambiguities in the measurements and several local optima, through a probabilistic formulation in a particle filter. The filter is reliably initialized through multiple attempts of an iterative optimization algorithm and implicitly selects the correct state 
hypothesis. We have proposed a sensor model which considers the Gaussian characteristics of the TDOA measurements at the receivers and explicitly takes into account measurement outliers for accurate and robust localization. In our motion model we assume a continuous movement of the signal beacon, which enables a quick recovery in case of temporary signal loss.

In experiments with our ultrasound system we have demonstrated the accuracy and reliability of our approach. The particle filter initialized successfully in less than 35 seconds in all experiments. In the subsequent tracking phase the algorithm localized the beacon with an average position error of less than $5 \mathrm{~cm}$ and was able to reliably recover the beacon position when it was lost due to signal blackouts. In experiments with random measurement outliers, which may occur from environmental influences such as noise and echoes, the particle filter proved to be hardly affected, even from very high errors of tenths of a second.

In our future work, we plan to extend our implementation to three dimensions and carry out further experiments localizing flying vehicles and smart phones.

\section{ACKNOWLEDGMENT}

This work has partly been supported by the German Research Foundation (DFG) within the Research Training Group 1103 (Embedded Microsystems). We thank Fabian Höflinger for his invaluable contribution in manufacturing the new hardware components.

\section{REFERENCES}

[1] M. L. Sichitiu and V. Ramadurai, "Localization of Wireless Sensor Networks with a Mobile Beacon," in Proceedings of the First IEEE Conference on Mobile Ad-hoc and Sensor Systems, 2004, pp. 174-183.

[2] P. Biswas and Y. Ye, "Semidefinite Programming for Ad Hoc Wireless Sensor Network Localization," in IPSN '04: Proceedings of the 3rd international symposium on Information processing in sensor networks. New York, NY, USA: ACM, 2004, pp. 46-54.

[3] B. Ferris, D. Hähnel, and D. Fox, "Gaussian Processes for Signa Strength-Based Location Estimation," in Proceedings of Robotics: Science and Systems Conference (RSS), 2006.

[4] M. Sippel, W. Kuntz, and L. Reindl, "New Approach in Precise Laser Tracking," in IEEE Instrumentation and Measurement Technology Conference Proceedings, 2008, pp. 446-451.

[5] N. B. Priyantha, A. Chakraborty, and H. Balakrishnan, "The Cricket Location-Support System," in MobiCom '00: Proceedings of the 6th annual international conference on Mobile computing and networking, 2000, pp. 32-43.

[6] A. Savvides, C. Han, and M. Strivastava, "Dynamic Fine-Grained Localization in Ad-Hoc Networks of Sensors," in Proceedings of the 7th annual international conference on Mobile computing and networking. ACM, 2001, pp. 166-179.

[7] W. Navidi, W. Murphy, and W. Hereman, "Statistical Methods in Surveying by Trilateration," Computational statistics \& data analysis, vol. 27, no. 2, pp. 209-227, 1998.

[8] L. Doherty, K. S. J. Pister, and L. E. Ghaoui, "Convex position estimation in wireless sensor networks," in INFOCOM 2001. Twentieth Annual Joint Conference of the IEEE Computer and Communications Societies. Proceedings. IEEE, vol. 3, 2001, pp. 1655-1663.

[9] S. Capkun, M. Hamdi, and J. P. Hubaux, "GPS-free positioning in mobile ad-hoc networks," in Proceedings of the 34th Annual Hawail International Conference on System Sciences, Jan. 2001, p. 10 pp.

[10] G. Calafiore, L. Carlone, and M. Wei, "A Distributed Gauss-Newton Approach for Range-based Localization of Multi Agent Formations," in 2010 IEEE International Symposium on Computer-Aided Control System Design (CACSD). IEEE, 2010, pp. 1152-1157.
[11] B. Xu, R. Yu, G. Sun, and Z. Yang, "Whistle: Synchronization-Free TDOA for Localization," in Distributed Computing Systems (ICDCS), 2011 31st International Conference on. IEEE, 2011, pp. 760-769.

[12] G. Shen, R. Zetik, and R. S. Thoma, "Performance Comparison of TOA and TDOA Based Location Estimation Algorithms in LOS Environment," in Positioning, Navigation and Communication, 2008. WPNC 2008. 5th Workshop on. Ieee, 2008, pp. 71-78.

[13] F. Gustafsson and F. Gunnarsson, "Positioning using time-difference of arrival measurements," in Acoustics, Speech, and Signal Processing, 2003. Proceedings.(ICASSP'03). 2003 IEEE International Conference on, vol. 6. IEEE, 2003, pp. VI-553.

[14] N. Ikoma, O. Tokunaga, H. Kawano, and H. Maeda, "Tracking of 3D Sound Source Location by Particle Filter with TDOA and Signal Power Ratio," in ICCAS-SICE, 2009. IEEE, 2009, pp. 1374-1377.

[15] J. Cho, H. Na, S. Kim, and C. Ahn, "Moving-Target Tracking Based on Particle Filter with TDOA/FDOA Measurements," ETRI Journal, vol. 34, no. 2,2012

[16] C. Hongyang, D. Ping, X. Yongjun, and L. Xiaowei, "A Robust Location Algorithm with Biased Extended Kalman Filtering of TDOA Data for Wireless Sensor Networks," in 2005 International Conference on Wireless Communications, Networking and Mobile Computing, 2005. Proceedings., vol. 2. IEEE, 2005, pp. 883-886.

[17] R. Zhang, F. Höflinger, and L. Reindl, "Tdoa based localization using interacting multiple model estimator and ultrasonic transmitter/receiver," in Systems, Signals and Devices (SSD), 2012 9th International MultiConference on, march 2012, pp. 1 -6.

[18] J. Saloranta and G. Abreu, "Solving the Fast Moving Vehicle Localization Problem via TDOA Algorithms," in Positioning Navigation and Communication (WPNC), 2011 8th Workshop on, april 2011, pp. 127 -130 .

[19] T. Janson, C. Schindelhauer, and J. Wendeberg, "Self-Localization Application for iPhone using only Ambient Sound Signals," in Proceedings of the 2010 International Conference on Indoor Positioning and Indoor Navigation (IPIN), Nov. 2010, pp. 259-268.

[20] C. Schindelhauer, Z. Lotker, and J. Wendeberg, "Network Synchronization and Localization Based on Stolen Signals," in Proceedings of 18th International Colloquium on Structural Information and Communication Complexity (SIROCCO), 2011.

[21] S. Thrun, "Affine Structure From Sound," in Proceedings of Conference on Neural Information Processing Systems (NIPS). Cambridge, MA: MIT Press, 2005.

[22] J. Wendeberg, T. Janson, and C. Schindelhauer, "Self-Localization based on Ambient Signals," Theoretical Computer Science, vol. 453, pp. 98 $109,2012$.

[23] H. Stewénius, "Gröbner Basis Methods for Minimal Problems in Computer Vision,” Ph.D. dissertation, Lund University, Apr. 2005.

[24] M. Pollefeys and D. Nister, "Direct computation of sound and microphone locations from time-difference-of-arrival data," in IEEE International Conference on Acoustics, Speech and Signal Processing, 2008. ICASSP 2008. IEEE, 2008, pp. 2445-2448.

[25] J. Wendeberg, F. Höflinger, C. Schindelhauer, and L. Reindl, "Anchorfree TDOA Self-Localization," in Indoor Positioning and Indoor Navigation (IPIN), 2011 International Conference on. IEEE, 2011, pp. $1-10$.

[26] R. Biswas and S. Thrun, "A Passive Approach to Sensor Network Localization," in Proceedings of the International Conference on Intelligent Robots and Systems, 2004. (IROS 2004). 2004 IEEE/RSJ, vol. 2, 2004, pp. 1544-1549.

[27] S. Thrun, W. Burgard, and D. Fox, Probabilistic Robotics. MIT Press, 2005.

[28] J. Kotecha and P. Djuric, "Gaussian particle filtering," IEEE Transactions on Signal Processing, vol. 51, no. 10, pp. 2592 - 2601, 2003.

[29] S. Thrun, D. Fox, W. Burgard, and F. Dellaert, "Robust monte carlo localization for mobile robots," Artificial Intelligence, vol. 128, no. 1-2, pp. 99-141, 2000.

[30] J. Liu, "Metropolized independent sampling with comparisons to rejection sampling and importance sampling," Statistics and Computing, vol. 6, 1996.

[31] K. Arun, T. Huang, and S. Blostein, "Least-Squares Fitting of Two 3-D Point Sets," Pattern Analysis and Machine Intelligence, IEEE Transactions on, no. 5, pp. 698-700, 1987.

[32] J. Liu and M. West, "Combined Parameter and State Estimation in Simulation-Based Filtering," in Sequential Monte Carlo Methods in Practice, A. Doucet, N. de Freitas, and N. Gordon, Eds., 2001, ch. 10. 